\title{
Fibroblastic Reticular Cell Tumor
}

National Cancer Institute

\section{Source}

National Cancer Institute. Fibroblastic Reticular Cell Tumor. NCI Thesaurus. Code C81758.

A very rare dendritic cell tumor affecting the lymph nodes, spleen, and soft tissues. Morphologically it is similar to the interdigitating dendritic cell sarcoma or follicular dendritic cell sarcoma. The tumor cells are positive for cytokeratin and CD68. Clinical outcome is variable. 